Recherches en didactique des langues et des cultures

Les cahiers de l'Acedle

15-3 | 2018

Quelles médiations en didactique des langues et des cultures?

Les tâches de médiation dans les manuels de Français Langue Étrangère pour le secondaire en Allemagne

Sílvia Melo-Pfeifer et Anna Schröder-Sura

(2) OpenEdition

Journals

Édition électronique

URL : http://journals.openedition.org/rdlc/3589

DOI : $10.4000 /$ rdlc.3589

ISSN : 1958-5772

Éditeur

ACEDLE

Référence électronique

Sílvia Melo-Pfeifer et Anna Schröder-Sura, "Les tâches de médiation dans les manuels de Français

Langue Étrangère pour le secondaire en Allemagne », Recherches en didactique des langues et des cultures [En ligne], 15-3 | 2018, mis en ligne le 01 septembre 2018, consulté le 20 avril 2019. URL http://journals.openedition.org/rdlc/3589; DOI : 10.4000/rdlc.3589

Ce document a été généré automatiquement le 20 avril 2019

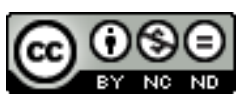

Recherches en didactique des langues et des cultures is licensed under a Creative Commons AttributionNonCommercial-NoDerivatives 4.0 International License 


\title{
Les tâches de médiation dans les manuels de Français Langue Étrangère pour le secondaire en Allemagne
}

\author{
Sílvia Melo-Pfeifer et Anna Schröder-Sura
}

\section{Introduction}

1 Depuis son entrée en force dans les documents officiels en Allemagne (Kultusministerkonferenz ou KMK, 2004, 2012), basés sur le Cadre Européen Commun de Référence pour les Langues (2001; dorénavant CECRL), la médiation linguistique (et culturelle) fait partie du socle de compétences à développer chez les élèves et à évaluer. En effet, la médiation linguistique, comme objet d'enseignement, constitue une partie importante des contenus présents dans les manuels de Langues Étrangères (LE). Elle constitue même, dans ces matériaux, la partie essentielle d'une des approches plurielles des langues et des cultures, la didactique intégrée, et légitime la réintroduction de la langue de l'école en classe de langues (Schröder-Sura \& Melo-Pfeifer, 2017).

Or, dans le CECRL, la compétence de médiation n'est, jusqu'alors' ${ }^{1}$, ni traitée de façon approfondie, contrairement à d'autres compétences plus établies, ni prise en considération à travers la définition de niveaux de compétence. Malgré l'absence de références structurées dans le CECRL, la médiation fait bel et bien son chemin, soit dans la recherche en Didactique des Langues (DL), soit en cours de LE, grâce notamment à son introduction dans les manuels (Reimann \& Rössler, 2013).

3 Dans cette contribution, nous nous attacherons à analyser les propositions de tâches de médiation présentes dans une série de manuels de Français Langue Étrangère (FLE) récemment publiés en Allemagne (A plus! Nouvelle édition). Par-delà cet exemple, nous chercherons à présenter la situation de la médiation en Allemagne : quelle est sa place 
dans le curriculum en général, et dans le secondaire 1 (Sekundarstufe I; de la $5^{\text {ème }}$ à la 10 ème classe) en particulier?

4 Nos questions de recherche quant à l'analyse des manuels sont les suivantes : Comment la compétence de médiation est-elle conçue dans ces manuels? Quelles situations de communication y sont présentées et quelles compétences mobilise-t-on? Entraine-t-on, dans ces scénarios, à un fonctionnement communicatif monolingue ou plurilingue? Quelles sont les démarches de correction et d'évaluation proposées pour les tâches de médiation? Comment les propositions de tâches évoluent-elles tout au long d'une série de manuels, du niveau A1 au niveau B2 ?

5 Notre analyse portera sur un éventuel filtrage de la définition et sur la manière dont la médiation a été opérationnalisée dans les manuels scolaires et proposera quelques pistes de réflexion sur le besoin de complexifier les approches de médiation déjà en place.

\section{Points de départ théoriques}

\section{Définitions de médiation en Didactique des Langues}

6 Le terme «médiation » connaît de multiples usages disciplinaires et sociaux (Araújo e Sá, De Carlo \& Melo-Pfeifer, 2014 ; North \& Piccardo, 2016; Piccardo, 2012 ; Stathopoulou, 2015 ; Zarate et al., 2003). Nous nous situons dans le champ de la DL, dans lequel le concept de médiation a connu une pertinence croissante après la publication du CECRL (2001). La médiation y est présentée comme une compétence à part entière, parmi d'autres compétences déjà traditionnellement ciblées en cours de LE. La médiation est définie comme le processus par lequel l'acteur social agit en tant qu'un « intermédiaire entre des interlocuteurs incapables de se comprendre en direct. Il s'agit habituellement (mais non exclusivement) de locuteurs de langues différentes»(Conseil de l'Europe, 2001: 71). L'objectif est clairement communicatif et ne vise pas la restitution exacte du message de départ (Hallet, $2008: 4$ ).

7 Plus récemment, selon l'optique choisie par Coste et Cavalli, «la notion de médiation prend une extension beaucoup plus importante, sans pour autant voir se dissoudre l'acception que proposait le CECRL ». Ils précisent: «par médiation, on désigne toute opération, tout dispositif, toute intervention qui, dans un contexte social donné, vise à réduire la distance entre deux (voire plus de deux) pôles altéritaires qui se trouvent en tension l'un par rapport à l'autre» (Coste \& Cavalli, 2015: 28). La médiation peut comprendre alors d'autres formes, en particulier la médiation cognitive et la médiation relationnelle : la médiation est alors cernée « soit comme visant l'accès à des informations et connaissances et la construction de compétences (médiation cognitive), soit comme contribuant à l'interaction, à la qualité de l'échange, à la résolution de conflits (médiation relationnelle) » (idem). Ces deux formes sont "souvent combinées » et "passent pour l'essentiel par une activité langagière de médiation (celle-là même, mais sensiblement étendue, que le CECRL avait définie) à l'intérieur de contextes sociaux » (Coste \& Cavalli, $2015: 13)$.

\section{Médiation et approches plurielles}

8 Il existe, en effet, un lien aisément explicitable entre la médiation et les approches plurielles des langues et des cultures. Par approches plurielles, nous entendons « des 
approches didactiques qui mettent en œuvre des activités d'enseignement-apprentissage qui impliquent à la fois plusieurs (= plus d'une) variétés linguistiques et culturelles » (Candelier et al., 2012: 6). La médiation et les approches plurielles contribuent conjointement au développement de la compétence plurilingue et pluriculturelle (voir aussi Bonvino, 2015 : 239). Puisque les activités de médiation mettent en jeu au moins deux langues qui font partie du cursus d'apprentissage, la médiation relève de la didactique intégrée des langues, qui constitue une des approches plurielles recensées par Candelier (2008), à côté de l'éveil aux langues, l'intercompréhension et les approches interculturelles dans leur diversité. De nombreux auteurs ont souligné les dimensions interculturelles de la compétence de médiation (cf. Hallet, 2008 ; Reimann, 2013 ; Zarate et al., 2003). Une étude de la relation complexe entre la médiation et l'intercompréhension a été entreprise par Bonvino (2015). Quoi qu'il en soit, il apparait clairement que des activités de médiation peuvent recourir à des démarches d'intercompréhension dans des buts de communication et d'apprentissage ${ }^{2}$. Un lien entre médiation et éveil aux langues semble plus difficile à établir car ce dernier ne prévoit généralement pas d'activités de réception ou de production.

De Pietro envisage l'élargissement des approches plurielles à la traduction, d'une part et à la communication exolingue, d'autre part (2014: 233-235). La traduction est une des variantes de la médiation citée par le CECRL (2001). Les concepts de médiation et de communication exolingue sont liés puisque, selon De Pietro (idem : 233), cette dernière « renvoie à des formes de communication entre des locuteurs qui, à des degrés divers, ne partagent pas les mêmes codes communicatifs et références culturelles $»^{3}$.

10 En pensant la médiation en lien avec les approches plurielles, on peut en tirer profit pour concevoir sa portée didactique et la mise en œuvre des descripteurs proposés par le Cadre de Références pour les approches plurielles des langues et des cultures (CARAP ; voir Candelier et al., 2012). Certains de ces descripteurs se réfèrent directement à la médiation dans ses aspects langagiers :

- Savoir rendre compte, dans une langue, d'informations traitées dans une ou plusieurs autres (S 6.4.1) ;

- Savoir varier/alterner les langues/les codes/les modes de communication (S 6.5.1);

- Engagement pour aider les personnes d'une autre culture/langue (A 8.6.3.1).

D'autres permettent d'expliciter, à un niveau didactique, certains aspects des médiations cognitive et relationnelle, telles que décrites par Coste et Cavalli (2015):

- savoir observer/analyser des éléments linguistiques/des phénomènes culturels dans des langues/ cultures plus ou moins familières (S 1);

- considérer/appréhender des phénomènes langagiers/culturels comme un objet d'observation/de réflexion (A 1.1.2);

- savoir communiquer en prenant en compte les différences sociolinguistiques/socioculturelles (S 6.3) ;

- volonté d'adaptation/de souplesse du comportement propre dans l'interaction avec des personnes linguistiquement/culturellement différentes de soi (A 13.1).

\section{La médiation dans les standards éducatifs en Allemagne}

Deux modèles de compétences pour l'enseignement/apprentissage des Langues Étrangères (LE), dans le sens où les apprenants ont le premier contact avec elles à l'école, ont été développés au cours des quinze dernières années, dans lesquels la médiation a été incluse dès le début. Dans le modèle le plus récent, publié pour le Secondaire II en 2012, valable désormais pour les LE de façon intégrée, la médiation - sous le terme de 
«Sprachmittlung $»^{4}$ - fait partie des compétences communicatives fonctionnelles à côté des quatre compétences traditionnelles compréhension/expression écrites et orales. Il s'agit de faire en sorte que les élèves " puissent restituer dans l'autre langue, tant à l'oral qu'à l'écrit, des aspects importants de textes oraux et écrits authentiques, pour un but donné et de façon adaptée au récepteur et à la situation, y compris pour des thèmes peu familiers » (KMK, $2012: 18$; notre traduction).

Les cinq compétences communicatives fonctionnelles sont en lien avec la compétence communicative interculturelle, la compétence relative aux textes et aux médias, ainsi qu'avec la conscience métalinguistique et la compétence d'apprentissage des langues (KMK, 2012:6).

13 Une partie de ces éléments se trouve déjà dans le modèle de compétences publié en 2003/2004 pour la première LE (anglais ou français) dans le Secondaire I - mais ils se trouvent structurés de façon un peu différente, moins complexe et moins intégrative (KMK, 2003/2004). Ici, la médiation est conçue de façon moins complexe: "les élèves peuvent transférer d'une langue vers l'autre langue le sens d'énoncés cohérents et de textes, oralement et par écrit » (KMK, 2003:67; notre traduction).

Ces modèles ouvrent la voie à divers formats de tâches de médiation dans les manuels, de façon particulièrement innovante et riche pour le Secondaire II (cf. Tesch, $2014:$ 17). Notons que la médiation, actuellement facultative, sera intégrée comme élément obligatoire de l'examen de baccalauréat à partir de l'année scolaire 2018/19.

\section{L'analyse de manuels : design méthodologique}

\section{Analyse des manuels scolaires de langues : retour sur un champ de recherche}

Le manuel scolaire, notamment dans le cadre de l'enseignement-apprentissage des LE, joue un rôle varié et assez riche : il est guide pédagogique, il présente une possible structuration des contenus, il propose des textes et progressions (pour les thèmes, pour la grammaire, pour le lexique, pour les contenus culturels et pragmatiques...). Il est à la fois un guide, voire un co-évaluateur des productions des apprenants, ainsi qu'un conseiller des démarches à suivre pour surmonter des difficultés (Puren, 2011).

À la suite des développements curriculaires suscités, d'un côté, par le CECRL et par la diffusion des approches plurielles, et, de l'autre côté, par les évolutions théoriques récentes en sociolinguistique et en DL, force est de constater que l'analyse des manuels, si elle était un peu démodée (et aussi critiquée que l'utilisation des manuels elle-même, Bertoletti, 1984), connaît un renouveau d'intérêt (Fäcke, 2016; Ferreira \& Melo-Pfeifer, 2015 ; Funk, 2004 ; Grünewald, 2011 ; Martinez, 2011 ; Morkötter, 2009 ; Schröder-Sura \& Melo-Pfeifer, sous presse). Ce retour sur l'analyse des manuels de LE est justifié par le désir de cerner le degré de diffusion de changement dans les pratiques scolaires (Fäcke, 2016), en fonction du rôle qu'y jouent les manuels.

Ainsi, l'analyse des manuels nous permet, de façon plus au moins tangible, de : i) mesurer la façon dont les nouvelles idées sont filtrées par les idéologies et les pratiques établies et se conjuguent avec elles; ii) cerner le poids de l'innovation pédagogique et didactique en classe; et, iii) comprendre comment ces innovations évoluent au fils du temps 
(simplifications, reconfigurations, abandon...). Nous n'approchons dans cette contribution que les deux premiers aspects.

C'est dans ce sens qu'il faut comprendre l'analyse que nous entreprenons ici. Le but de notre recherche n'est pas d'examiner de façon critique un manuel donné, mais bien de cerner, sur un exemple particulier, d'une part, l'état des lieux de l'introduction des tâches de médiation dans les manuels en Allemagne et, d'autre part, la façon dont la définition de « médiation » a été filtrée et prise en compte. Il faut, de plus, souligner que les manuels de LE en Allemagne sont pionniers dans l'introduction de tâches de médiation, encore très rares dans d'autres contextes.

\section{Méthodologie de l'analyse}

Pour notre étude, nous avons mené une analyse quantitative et qualitative des tâches signalées par les auteurs des manuels comme contribuant à la «compétence de médiation ». Pour une définition opératoire de "tâche de médiation", nous nous appuyons sur Stathopoulou (2015:62) :

Mediation tasks are those tasks which require learners to relay information from one language to another for a given communicative purpose. (...). Specifically, when language users are asked to complete a mediation task, they first have to understand the information included in the text of a given language and then relay some of its messages to another language in a way that is appropriate to the context of situation. In other words, they are required to select which messages serve the purpose of a specific communicative encounter so as to transfer them appropriately and accurately ${ }^{5}$ (Stathopoulou, 2015: 62).

Pour ce qui est de l'analyse quantitative, qui est le point central de cette contribution, nous avons décompté les activités proposées relevant de la médiation, suivant une grille d'analyse que nous avons construite à partir de Caspari (2013), dans le but de répondre aux questions de recherche présentées dans l'introduction.

Dans un premier temps, nous avons distribué les tâches par rapport à la modalité de production (orale ou écrite) et à la langue dans laquelle il faudrait accomplir la tâche (FLE ou allemand langue maternelle/seconde langue).

Dans un second temps, nous avons réparti les tâches par rapport à leur nature et leurs objectifs (Tableau 1).

Tableau 1 - Catégories d'analyse : les tâches de médiation (adapté de Caspari, 2013 : 36)

\begin{tabular}{|l|l|}
\hline \multirow{4}{*}{ Production orale } & Contrôle de la compréhension orale/écrite (en LM/LS $)$ \\
\cline { 2 - 3 } & Préparation à une tâche dans la LE \\
\cline { 2 - 3 } & Paraphrase/Résumé (en FLE) \\
\cline { 2 - 3 } & Transfert d'actes de parole dans une situation d'interaction \\
\hline \multirow{3}{*}{ Production écrite } & Contexte pour la production écrite \\
\cline { 2 - 3 } & Contrôle de connaissances lexicales et grammaticales \\
\cline { 2 - 3 } & Utilisation de connaissances interculturelles \\
\cline { 2 - 2 } &
\end{tabular}


Résumé de texte (oral ou écrit) - Contrôle de la compréhension

Compilation d'informations pertinentes

\section{sont révélées insuffisantes pour rendre compte de l'évolution des tâches, notamment en} ce qui concerne les langues de réception et de production visées.

24 l'évolution de la perception de la médiation en rapport avec les niveaux de compétences.

\section{Analyse du manuel « À Plus ! Nouvelle édition »}

\section{Distribution et évolution des tâches de communication}

«À plus! Nouvelle édition » (Französisch für Gymnasien/Français pour le Lycée) est une série de quatre manuels de FLE, publiée entre 2012 et 2015, en Allemagne, par la maison d'édition Cornelsen (Blume et al., 2012, 2013 et 2014 ; Gregor et al., 2015), du niveau A1 au niveau B2. Une première observation de l'index des manuels (version élève) nous laisse constater que :

- les tâches de médiation ont un statut assez élevé, puisque les manuels incluent des symboles s'y référant, en pied d'égalité avec l'interculturel, par exemple ;

- les unités ne disposent pas toutes de tâches ou d'exercices de médiation ;

- il existe des unités avec plusieurs tâches de médiation (jusqu'à 4 : ex. Unité 6, A Plus !1);

- les tâches de médiation sont souvent, mais pas toujours, incluses dans «Fais le point » et sont parfois facultatives;

- les manuels incluent des conseils sur comment accomplir des tâches de médiation dans la section « Méthodes » (ex. À Plus ! 4, p. 133).

Par rapport aux tâches proposées dans les quatre volumes, le tableau 2 permet de cerner une stabilité relative dans la prise en charge de ces tâches tout au long de la série :

Tableau 2 - Aperçu général de la présence des tâches de médiation dans les quatre manuels

\begin{tabular}{|l|l|l|l|l|}
\hline \multicolumn{4}{|c|}{ Nombre de tâches par volume } & \multirow{2}{*}{ Total } \\
\cline { 1 - 4 } À Plus! 1 & À Plus ! 2 & À Plus ! 3 & À Plus ! 4 & \\
\hline 8 & 7 & 9 & 12 & 36 \\
\hline
\end{tabular}

27 En ce qui concerne les situations communicatives, nous remarquons qu'elles sont conçues, pour ce qui est du " produit discursif cible », en termes de production à l'oral ou à l'écrit (Tableau 3).

Tableau 3 - Distribution des tâches de médiation

\begin{tabular}{|l|l|l|l|l|l|l|}
\hline & & À Plus !1 & À Plus ! 2 & À Plus ! 3 & À Plus ! 4 & Total \\
\hline
\end{tabular}




\begin{tabular}{|l|l|l|l|l|l|l|}
\hline \multirow{2}{*}{ Médiation \& production orale } & En LM/LScol & $7(1)$ & $5(1)$ & 4 & $4(1)$ & $20(3)$ \\
\cline { 2 - 8 } & En FLE & 1 & $2(1)$ & 3 & 5 & $11(1)$ \\
\hline \multirow{2}{*}{ Médiation \& production écrite } & En LM/LScol & 0 & 0 & 1 & 1 & 2 \\
\cline { 2 - 8 } & En FLE & 0 & 0 & 1 & 2 & 3 \\
\hline Total & & $8(1)$ & $7(2)$ & 9 & $12(1)$ & $36(4)$ \\
\hline
\end{tabular}

Ce tableau nous montre que, en situation de production orale, c'est la production en langue maternelle/langue de scolarisation (LM/LS) qui prédomine. De plus, la production en LE est assez rare et quasiment réservée aux niveaux plus avancés, et la production écrite est absente dans les deux premiers volumes. Force est aussi de constater que le nombre de tâches dans lesquelles les apprenants doivent produire en LM/LS diminue de façon constante tout au long de la série, ce qui peut signifier que la place de la LM (langue seconde et de scolarisation pour un nombre croissant d'élèves issus de l'immigration), bienvenue dans les niveaux d'initiation, perd progressivement sa valeur.

\section{Typologie des tâches de médiation}

Nous avons également souhaité déceler la nature des tâches proposées, afin de comprendre quels buts leur sont attachés. Le tableau 4 illustre les résultats de l'analyse, ainsi que l'évolution des tâches proposées.

Tableau 4 - Nature des tâches de médiation (adapté de Caspari, 2013 : 36)

\begin{tabular}{|c|c|c|c|c|c|c|}
\hline & & $\begin{array}{l}\grave{A} \text { Plus! } \\
1\end{array}$ & $\begin{array}{l}\grave{A} \\
2\end{array}$ & $\begin{array}{ll}\text { À } & \text { Plus ! } \\
3 & \end{array}$ & $\begin{array}{ll}\grave{A} & \text { Plus ! } \\
4\end{array}$ & Total \\
\hline \multirow{4}{*}{$\begin{array}{l}\text { Production } \\
\text { orale }\end{array}$} & $\begin{array}{l}\text { Contrôle de la compréhension orale/ } \\
\text { écrite (en LM/LS) }\end{array}$ & 6 & 5 & 4 & $2\left(2^{*}\right)$ & 17 \\
\hline & Préparation à une tâche dans la LE & 0 & 0 & 0 & 2 & 2 \\
\hline & Paraphrase/Résumé (en FLE) & & 1 & 3 & 4 & 8 \\
\hline & $\begin{array}{l}\text { Transfert d'actes de parole dans une } \\
\text { situation d'interaction }\end{array}$ & 2 & 2 & 0 & 1 & 5 \\
\hline \multirow{3}{*}{$\begin{array}{l}\text { Production } \\
\text { écrite }\end{array}$} & Contexte pour la production écrite & 0 & 0 & 0 & 1 & 1 \\
\hline & $\begin{array}{l}\text { Contrôle de connaissances lexicales } \\
\text { et grammaticales }\end{array}$ & 0 & 0 & 0 & $\left(1^{*}\right)$ & 0 \\
\hline & $\begin{array}{l}\text { Utilisation de connaissances } \\
\text { interculturelles }\end{array}$ & 0 & 0 & 0 & 1 & 1 \\
\hline
\end{tabular}




\begin{tabular}{|l|l|l|l|l|l|l|l|}
\hline \begin{tabular}{|l|l|l|l|} 
Résumé de texte (oral ou \\
écrit) - Contrôle de la \\
compréhension
\end{tabular} & 0 & 0 & $(1)$ & 1 & $1(1)$ \\
\hline $\begin{array}{l}\text { Compilation d'informations } \\
\text { pertinentes }\end{array}$ & 0 & 0 & $1(1)$ & 0 & $1(1)$ \\
\hline Total & 8 & 8 & $8(2)$ & 12 & $\begin{array}{l}36 \\
(2)\end{array}$ \\
\hline
\end{tabular}

Nous observons que, dans la majorité des tâches proposées, il s'agit de contrôler la compréhension orale ou écrite, l'apprenant devant expliciter, en LM/LS, ce qu'il a compris de la lecture ou de l'écoute des documents. On pourrait dire, en effet, que la production d'une paraphrase ou d'un résumé relève aussi du contrôle de la compréhension. Nous avons voulu garder ces deux tâches séparément, puisque, dans le cas du résumé et de la paraphrase, on fait exprès, soit de demander ce type de production spécifique, soit de demander de le faire dans la LE. Étant donné le nombre assez réduit de tâches de production écrite, il n'est pas possible d'en déduire des tendances.

31 Si l'on considère les situations de communication scénarisées (bien que le statut du contexte semble souvent peu pertinent), nous remarquons qu'on a affaire à des situations communicatives exolingues (déséquilibre de ressources/répertoires linguistiques en présence). La médiation est ainsi conçue en tant que compétence ou mode de communication interlinguistique (la médiation intralinguistique, que ce soit dans la LM/ LS ou la LE n'étant pas incluse). Dans ces cas de communication exolingue, l'élève est mis en position d'expert, le seul expert d'ailleurs, sans autres ressources que ses savoirs; par contre, son interlocuteur est mis en situation d'incompréhension totale ou partielle, à l'aide de formulations comme : « il ne comprend pas très bien » (Figure 1).

Figure 1 - Exercice extrait de $\grave{A}$ Plus 11 (p. 133)

\section{Médiation}

2 Tu es avec un ami dans une boulangerie en France. Deux jeunes sont devant vous.

CD2 a Écoute le dialogue entre les jeunes et la vendeuse. Ton ami ne comprend pas le français. Fais la médiation. Hör dir das Gespräch zwischen den Jugendlichen und der Verkäuferin an. Erkläre deinem Freund, der kein Französisch versteht, was die Jugendlichen kaufen.

b Travaillez à deux. Écoutez le dialogue encore une fois et trouvez comment on dit. Le partenaire A écoute la vendeuse, le partenaire B écoute les deux jeunes.

$$
\text { - }
$$$$
-
$$

Généralement, il s'agit de faire la médiation entre un interlocuteur natif de la LE (ou d'un document authentique en LE) et un natif de la LM/LS ce qui, encore une fois, n'épuise pas les contextes dans lesquels on est appelé à faire la médiation dans la vie réelle: la médiation peut être faite dans une situation exolingue / endolingue où tous les interlocuteurs parlent le français comme LE, par exemple.

Un regard sur les tâches communicatives permet d'observer que les instructions sont souvent vagues, stéréotypées et pouvant induire des ambiguiités, surtout en ce qui concerne la langue à utiliser. Un exemple assez intéressant concerne l'accueil d'une «corres » [sic.] (correspondante) étrangère à la maison, où la langue à utiliser pour 
expliquer la grille télévisée n'est pas explicitée : faut-il pratiquer la langue-cible de celui qui accueille (le français pour l'étudiant allemand) ou la langue-cible de celui qui fait la mobilité (l'allemand, dans notre cas) ? Ou bien les deux, mettant en place des stratégies d'intercompréhension interfamiliale? Nous avons pu constater qu'il est trop rare de trouver des tâches de médiation où l'on incite la pratique de comportements bi-/ plurilingues communs dans des situations de communication exolingue.

En observant les démarches d'évaluation proposées, nous remarquons qu'elles sont assimilables à des tâches de traduction, impliquant le plus souvent le passage du discours direct au discours indirect. L'exemple dans le Tableau 5 rend compte de cette constatation. La tâche consiste à lire un e-mail reçu de Flore, de France, et à raconter à une amie qui ne parle pas le français ce que l'elle a compris.

Tableau 5 - Exemple de démarche de tâche et de correction proposée (À Plus ! 1, p. 19)

\begin{tabular}{|l|l|}
\hline Énoncé original & Solution proposée \\
\hline $\begin{array}{l}\text { Bonjour, je m'appelle Flore! Je suis de } \\
\text { Colmar. C'est en Alsace, près de Strasbourg. Je } \\
\text { suis nouvelle à Lille. C'est dans le Nord de la } \\
\text { France. Je suis en sixième, à l'école « Émile } \\
\text { Zola » et ça va super bien. }\end{array}$ & $\begin{array}{l}\text { Flore ist aus Colmar. Das ist in der Nähe von } \\
\text { Straßburg. Sie ist neu in Lille und geht in die } \\
\text { sechste Klasse der Schule Émile Zola. Dort ist es } \\
\text { Euper. Sie fragt, wie es mir geht und ob ich auch } \\
\text { Et toi, ça va? Tu es aussi en sixième? }\end{array}$ \\
$\begin{array}{l}\text { Salut et à plus, } \\
\text { Flore }\end{array}$ & \\
\hline
\end{tabular}

Une autre tâche consiste à lire une brochure informative sur un parc et à répondre aux questions « de la classe » (Figure 2). Il s'agit d'un entraînement à une lecture repérage où il faut identifier des informations spécifiques. 


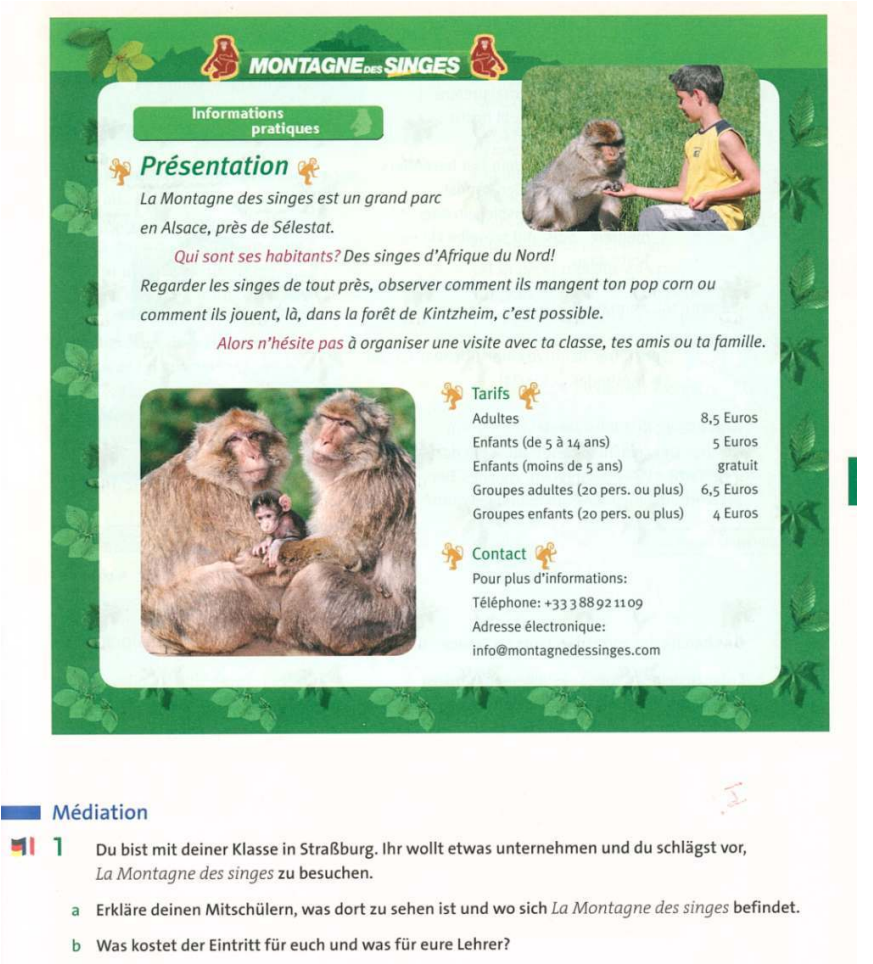

En regardant le type d'activité et la correction proposée, nous constatons qu'il s'agit de questions et réponses plutôt «typiques » d'un contrôle de la compréhension écrite en classe de LE, et que les signes d'interaction sont assez rares (par exemple : on n'est pas sûr-e-s que les collègues aient compris l'information parce qu'on ne trouve jamais de stratégies de contrôle de la compréhension d'autrui). La dimension métalinguistique et métacommunicative, qu'on aurait intérêt à associer dès le début aux tâches de médiation, est donc absente : les apprenants-médiateurs ne font jamais un travail d'explicitation des stratégies de compréhension à leurs interlocuteurs.

\section{Synthèse}

Les tâches de médiation qui apparaissent dans les manuels analysés sont en rapport avec les définitions de "médiation" des documents régulateurs en Allemagne. Elles contribuent à un renouveau didactique en cours de LE, notamment par la légitimation de l'entrée de la LM/LS dans les cours. Ces tâches envisagent une proximité avec des situations réelles de communication et portent un intérêt renouvelé aux documents dits "authentiques». Nous reconnaissons qu'en contexte de salle de classe, de par les contraintes qui le caractérisent, il est difficile de tout intégrer et de tout didactiser; les manuels de LE font un travail de sélection des contenus, des tâches, des approches et des ingrédients du contexte censés être les plus pertinents, pour construire une progression cohérente. Pour la progression, nous constatons une complexification progressive. D'une part, un passage de la médiation comme tâche visant la production orale à une tâche visant la production écrite; d'autre part, un passage de cette production de la LM ou LS à la LE. 
Dans ce qui suit, nous précisons trois tendances dans la mise en œuvre de la médiation en tant que tâche scolaire, repérées grâce à l'analyse des manuels.

\section{Transfert d'informations d'une langue à l'autre}

La première tendance est une appréhension encore partielle de la médiation, déjà reconnue par North \& Piccardo (2016). Ces auteurs s'étonnent de la réduction des mondes possibles ouverts par les tâches de médiation dans l'enseignement-apprentissage des langues, qui sont presque toujours réduits au transfert d'informations d'une langue à l'autre (plus que d'un acteur social à l'autre) :

Where mediation has been included in curricula and examinations, it tends to involve informal interpretation/translation or summary of a text - written or spoken - in one language into another language. Why has this interpretation prevailed ?7 (North \& Piccardo, $2016: 6$ ).

Ils reconnaissent, cependant, que la formulation même du CECRL, ainsi que les liens établis avec les tâches déjà connues (voire canoniques), telles que le résumé ou la traduction, ont facilité une appréhension stéréotypée des tâches de médiation. Ceci dit, il faudrait donc complexifier les transpositions didactiques à partir des consignes présentes dans les matériaux proposés. Cette complexification permettrait de saisir la médiation dans toute sa complexité :

Mediation is concerned with the role of language in processes like the creation of the space and conditions to facilitate communication, understanding and/or learning, the construction and co-construction of new meaning, and/or the conveyance of information ${ }^{8}$. (North \& Piccardo, $2016: 15$ ).

\section{La médiation instrumentalisée et minorée/accessoire}

41 La seconde tendance se réfère à une position (encore) accessoire de la compétence de médiation par rapport aux compétences (ou "modes de communication », comme le suggèrent North \& Piccardo, 2016) plus établies. Ainsi, nous avons pu cerner que la médiation est souvent instrumentalisée au service du contrôle de la compréhension, le plus souvent écrite, de textes (pseudo-)authentiques par l'usage de la LM ou LS (seul le tandem linguistique français-allemand est admis dans les tâches). De plus, les tâches de médiation ont parfois un statut optionnel, elles ne sont pas incluses dans toutes les unités et les critères de correction et d'évaluation sont souvent peu spécifiques.

\section{Une conception restreinte de la médiation}

42 Notre analyse a permis de repérer des aspects à développer ou à approfondir dans le format des tâches de médiation et qui sont liés à une conception peu souple de la médiation, diminuant ainsi le pouvoir de cette notion et son potentiel de "porteur de développements " (Piccardo, 2012: 286). Nous regroupons nos analyses autour de cinq aspects: i) la vision simpliste et essentialiste de la communication plurilingue et interculturelle; ii) la vision partielle de la compétence stratégique ; iii) le paradoxe des modalités de résolution des tâches de médiation; iv) la présentation stéréotypée des situations, des locuteurs et du rôle de l'apprenant; et, pour finir, v) la visée monolingue et monoglossique de ces tâches. 


\section{(i) Une vision simpliste et essentialiste de la communication plurilingue et interculturelle}

Force est de constater que la médiation est toujours associée à des problèmes de communication linguistique, comme si le manque de compétences linguistiques était le seul obstacle à la communication exolingue. Si nous pensons aux rôles du médiateur inventoriés par Dendrinos (2006) - moniteur du processus d'interaction, facilitateur des échanges exolingues, négociateur de sens en cas de conflit et arbitre/compromissoire nous repérons bien dans les manuels une tendance à n'évoquer que le rôle de "facilitateur des échanges exolingues ». Or, on le sait, la médiation ne doit pas être comprise exclusivement dans le sens de transmission d'une information d'une langue à l'autre et d'un interlocuteur à l'autre dans des environnements communicatifs aseptiques et minimalistes, mais elle suppose la prise en compte de la diversité de situations où l'on co-construit le sens de l'interaction dans l'interaction avec et entre des acteurs sociaux. Comme tâche sociale complexe, la médiation, telle qu'elle est pratiquée en cours, devrait entraîner à la complexité des rapports individuels, sociaux, culturels et linguistiques.

\section{(ii) Une vision partielle de la compétence stratégique}

44 Les tâches analysées reposent sur une vision linguiciste de la communication qui est marquée par une absence d'indications sur l'usage du para- et du non-verbal, l'usage de la langue étant le point de départ et d'arrivée des tâches. Ainsi, la résolution de problèmes de communication et le transfert d'un message se font exclusivement par la voie «verbale» de la communication. Il faut aussi dire que notre usage de «langue » au singulier n'est pas innocent dans ce contexte (voir point v) : il s'agit, en effet, de mobiliser un code linguistique spécifique (en l'absence de toute information sur d'autres répertoires linguistiques partagés).

\section{(iii) Le paradoxe des modalités de résolution des tâches de médiation}

45 Ce paradoxe consiste à demander à l'apprenant de réaliser seul ce qui devrait être une tâche (fondamentalement) communicative. Les situations sont souvent présentées comme interactionnelles (et souvent synchrones), mais l'élève doit « agir » tout seul en classe, sans prendre compte des réactions, à la fois verbales et non-/para-verbales de ses interlocuteurs. On y retrouve une vision non socioconstructiviste, puisque la coconstruction du sens avec un interlocuteur en est absente.

\section{(iv) Une présentation stéréotypée des situations, des locuteurs et du rôle de l'apprenant}

Le caractère stéréotypé des tâches de médiation est visible à travers la simplification, stabilisation et réduction du contexte, de l'interlocuteur et du rôle de l'apprenant. En effet, le contexte de communication est souvent présenté avec un scénario minimal et avec peu d'informations caractérisant la médiation comme une tâche également interculturelle. De son côté, l'interlocuteur et son rôle de co-agent sont généralement peu visibles. Les questions de rapports de pouvoir et du pouvoir du médiateur restent peu apparentes. L'apprenant-médiateur lui-même est un simple expert de la langue (ou mis en position d'expertise), souvent sans intentions (sauf celle de transmettre un message), 
dont les éventuels conflits et affects ne sont pas pris en compte. On passe ainsi sous silence les questions éthiques de la médiation (médiation et manipulation, médiation et pouvoir, médiation et gestion des faces, etc.) (cf. Dendrinos, 2006:12; pour un exemple d'activité mettant en place des échanges reposant sur de véritables enjeux, cf. De FlorioHansen, 2013: 84-85). Dendrinos (2006) explique clairement cet aspect éthique de la médiation par rapport à la question du pouvoir du médiateur : le médiateur a souvent le pouvoir de choisir des informations à transmettre, la perspective à adopter, les arguments à évoquer. C'est bien l'éthique de la communication humaine dans le sens large qui devrait être mise en scène dans les tâches de médiation. Penser la médiation en cours de LE et l'introduire en tant que tâche de communication devraient offrir l'occasion de penser la dimension éthique de l'enseignement-apprentissage des langues, bien audelà des compétences stratégiques utilitaires (Castellotti, 2017).

\section{(v) Une visée monolingue et monoglossique des tâches}

47 Si nous considérons que les cours de LE doivent viser le développement de la compétence plurilingue (Schädlich, 2016), alors il faut remarquer que la médiation telle qu'elle est conçue comme tâche pédagogique dans les manuels analysés reste au service exclusif de la langue enseignée. Elle n'est pas vue comme moyen de développement de la compétence plurilingue et interculturelle dans sa globalité ni comme moyen de prise en compte de l'ensemble du répertoire plurilingue de chaque interlocuteur (voir Araújo e Sá, De Carlo \& Melo-Pfeifer, 2014, pour le fonctionnement de la médiation dans des interactions plurilingues médiatisées par ordinateur). Elle semble encore assez éloignée d'une préparation à des situations concrètes/réelles de médiation où l'on mobilise toutes les ressources sémiotiques dont on dispose pour co-construire le sens (une véritable pratique translangagière, comme le réfère Stathopoulou, 2014: 40) et où l'élève semble encore éloigné d'un acteur social plurilingue, ayant fait l'expérience de plusieurs langues et cultures. Quant à l'usage des langues, un deuxième paradoxe pourrait être nommé : bien que la médiation, telle qu'elle est (partiellement) mise en place en cours de LE, soit une tâche à nature bilingue, puisqu'elle fait appel à deux langues en cours, elle privilégie des productions monolingues (production et réception dans des langues séparées) et sans traces d'hybridation linguistique du discours. Cette façon de présenter la médiation a tendance, corolairement, à avantager une vision du médiateur bilingue comme un double monolingue et, surtout dans des cours plus avancés, comme un double monolingue " équilibré " qui se rapproche, peu à peu, du locuteur natif. Ainsi, la médiation, qui pourrait être un outil pour relativiser les idéologies monolingues et monoglossiques en cours de LE, peut, paradoxalement, aider à les soutenir.

\section{Perspectives}

Prenant appui sur les tendances diagnostiquées et afin de cibler une vision plus réaliste de la médiation en tant que tâche sociale normale dans la vie quotidienne, il faudrait, à notre avis, inclure une plus grande diversité de tâches et d'autres tâches encore absentes ou assez rares comme celles en rapport, d'une part, avec la médiation interculturelle et, d'autre part, avec la médiation plurilingue, où l'on admet des géométries variables des répertoires individuels et où l'on présente plus que des dyades linguistiques (français et allemand, dans le cas de cette étude). Ces dernières auraient l'avantage de traiter et de développer des stratégies de communication bi-/plurilingues. 
Pour finir, nous prônons le besoin d'un appui sur des descriptions linguistiques et pragmatiques du fonctionnement de pratiques discursives « réelles » de médiation en situations de communication exolingues (non seulement monolingues mais aussi plurilingues). Ceci permettrait: i) une meilleure didactisation des situations de médiation, à l'aide de tâches pédagogiques plus concrètes et holistiques ; ii) la conception de critères de correction et d'évaluation accordant moins d'importance à la norme linguistique dans la langue-cible; iii) l'élaboration de descripteurs d'évaluation moins mécaniques et répétitifs plus aptes à rendre compte de la diversité des tâches, des compétences, des buts et des moyens mis en scène pendant les tâches de médiation. Pour cela, il faudrait que les textes règlementaires eux-mêmes - en particulier pour le Secondaire I - soient reformulés dans ce sens, en tenant compte des compétences dont disposent les apprenants à ce niveau, dans l'ensemble de leurs répertoires, et qu'il convient d'exploiter dans toute leur diversité.

On aura reconnu, dans les lignes ci-dessus, de nombreux aspects qui relèvent de ce que Coste et Cavalli (2015) entendent par médiation relationnelle. Le chemin qu'il reste à faire concerne également ce qui correspond à la médiation cognitive. Il serait souhaitable que la médiation elle-même, ainsi que les mises en lien interlinguistiques auxquelles elle donne lieu, fasse l'objet de démarches métacommunicatives et métalinguistiques réflexives. C'est dans ce sens que plaide également Königs (2015: 38 ; pour un exemple d'activité, cf. De Florio-Hansen, $2013: 80$ ).

\section{BIBLIOGRAPHIE}

Araújo e Sá, Mª H., De Carlo, M. \& Melo-Pfeifer, S. (2014). « Acteurs et dynamiques de médiation dans une plateforme de formation à l'Intercompréhension ». Revue Canadienne des Langues Vivantes/The Canadian Modern Language Review, vol. 70, n⒉ pp. 133-157.

Bertoletti, M. C. (1984). « Manuels et matériels scolaires pour l'apprentissage du F.L.E. Ébauche d'une grille d'analyse ». Le Français dans le Monde, vol. 186, pp. 55-63. Consulté en mars 2017 : http://www.christianpuren.com/biblioth\%C3\%A8que-de-travail/032/.

Blume, O.-M., Gregor, G., Jorißen, C. \& Mann-Grabowski, C. (2012). À plus ! 1 Nouvelle édition. Französisch für Gymnasien (livre de l'élève). Berlin : Cornelsen.

Blume, O.-M., Gregor, G., Jorißen, C. \& Mann-Grabowski, C. (2013). À plus ! 2 Nouvelle édition. Französisch für Gymnasien (livre de l'élève). Berlin : Cornelsen.

Blume, O.-M., Gregor, G., Jorißen, C., \& Mann-Grabowski, C. (2014). À plus ! 3 Nouvelle édition. Französisch für Gymnasien (livre de l'élève). Berlin : Cornelsen.

Bonvino, E. (2015). « Sprachmittlung und Interkomprehension : ist ein Dialog möglich ?». In Nied Curcio, M., Katelhöm, P. \& Bašić, I. (dir.), Sprachmittlung - Mediation - Mediazione Linguistica.

Berlin : Frank \& Timme. pp. 231-242. 
Candelier, M. (2008). « Approches plurielles, didactiques du plurilinguisme : le même et l'autre ». Cahiers de l'ACEDLE, vol. 5. pp. 65-90. Consulté en juillet 2017 : https://acedle.org/old/spip.php? article1009.

Candelier, M., Camilleri-Grima, A., Castellotti,V., de Pietro, J.-F., Lörincz, I., Meißner, F.-J., Schröder-Sura, A., Noguerol, A. \& Molinié, M. (2012). Le CARAP - Un Cadre de Référence pour les Approches plurielles des langues et des cultures-Compétences et ressources. Strasbourg: Conseil de l'Europe. Consulté en mars 2017 : http://carap.ecml.at/.

Candelier, M. \& Schröder-Sura, A. (2015). « Les approches plurielles et le CARAP : origines, évolutions, perspectives ». In Pietro, J.-F. \& Gerber, B. (dir.), Les approches plurielles des langues et des cultures, Babylonia, vol. 2. pp. 12-19.

Caspari, D. (2013). « Sprachmittlung als kommunikative Situation. Eine Aufgabentypologie als Anstoß zur Weiterentwicklung eines Sprachmittlungsmodells ». In Reimann, D. \& Rössler, A. (dir.), Sprachmittlung im Fremdsprachenunterricht. Tübingen : Narr. pp. 27-43.

Castellotti, V. (2017). Pour une didactique de l'appropriation. Diversité, compréhension, relation. Paris : Les Éditions Didier.

Coste, D. \& Cavalli, M. (2015). Education, mobilité, altérité. Les fonctions de médiation de l'école. Strasbourg : Conseil de l'Europe. Consulté en mars $2017:$ http://www.coe.int/t/dg4/Linguistic/ Source/LE_texts_Source/LE\%202015/Education-Mobility-Otherness_en.pdf.

Conseil de l'Europe (2001). Cadre européen commun de référence pour les langues. Strasbourg : Conseil de l'Europe.

De Florio-Hansen, I. (2013). « Sprachmittlung in alltagsweltlicher Kommunikation. Eine komplexe Herausforderung für Fremdsprachenlehrer und -lerner ». In Reimann, D. \& Rössler, A. (dir.), Sprachmittlung im Fremdsprachenunterricht. Tübingen: Narr. pp. 65-92.

Dendrinos, B. (2006). « Mediation in communication, language teaching and testing ». Journal of Applied Linguistics, vol. 22. pp. 9-35.

De Pietro, J.-F. (2014). « Des approches plurielles, oui, mais combien?». In Troncy, Ch. (Dir), de Pietro, J.-F., Goletto, L. \& Kervran, M. (Colls) (2014), Didactique du plurilinguisme - Approches plurielles des langues et des cultures - Autour de Michel Candelier. Rennes : PUR. pp. 227-238.

Fäcke, Ch. (2016). « Lehrwerkforschung - Lehrwerkgestaltung Lehrwerkrezeption Überlegungen zur Relevanz von Lehrwerken für den Fremdsprachenunterricht. In Rückl, M. (dir.), Sprachen und Kulturen : vermitteln und vernetzen Beiträge zu Mehrsprachigkeit und Inter-/Transkulturalität im Unterricht, in Lehrwerken und in der Lehrer/innen/bildung. Münster : Waxmann. pp. 34-48.

Ferreira, T. \& Melo-Pfeifer, S. (2015). « Desenvolvimento da competência plurilingue : quebrar o habitus monolingue em manuais de língua ». In Araújo e Sá, M. H. \& Pinho, A. (dir.) Intercompreensão em contexto educativo : resultados da investigação. Aveiro : Universidade de Aveiro. pp. 133-156.

Funk, H. (2004). « Qualitätsmerkmale von Lehrwerken prüfen - ein Verfahrensvorschlag ». Babylonia, vol. 3, nº 04/5. pp. 41-47.

Gregor, G., Jorißen, C., Mann-Grabowski, C., Nikolic, L., Philipp, D.-W., Raliarivony-Freytag, F. \& Wagner, E. (2015). À plus! 4 Nouvelle édition. Französisch für Gymnasien (livre de l'élève). Berlin : Cornelsen.

Grünewald, A. (2011). « Förderung der Interkulturellen Kompetenz in Französisch- und Spanischlehrwerken ». Fremdsprachen Lehren und Lernen. Themenschwerpunkt : Lehrwerkkritik, Lehrwerkverwendung, Lehrwerkentwicklung, vol. 2. pp. 64-82. 
Hallet, W. (2008). Zwischen Sprachen und Kulturen vermitteln. Interlinguale Kommunikation als Aufgabe ». Der Fremdsprachliche Unterricht Englisch, 93. pp. 2-7.

Königs, F. G. (2015). « Sprachen lernen - Sprachen mitteln : Warum das eine nicht ohne das andere geht ». In Nied Curcio, M., Katelhön, P. \& Bašić, I. (dir.), Sprachmittlung - Mediation Mediazione Linguistica. Berlin : Frank \& Timme. pp. 29-40.

Kultusministerkonferenz (2004). Bildungsstandards für die erste Fremdsprache (Englisch/Französisch) für den mittleren Schulabschluss. Beschluss vom 04.12.2003. München : Wolters Kluwer.

Kultusministerkonferenz (2012). Bildungsstandards für die fortgeführte Fremdsprache (Englisch/ Französisch) für die allgemeine Hochschulreife. Beschluss vom 18.10.2012. Beschlüsse der Kultusministerkonferenz. Consulté en mars 2017 : http://www.kmk.org/fileadmin/Dateien/ veroeffentlichungen_beschluesse/2012/2012_10_18-Bildungsstandards-Fortgef-FS-Abi.pdf.

Martinez, H. (2011). « Kompetenzorientierung und Lehrwerke für Französisch und Spanisch : Zwischen Tradition und Innovation ». Fremdsprachen Lehren und Lernen. Themenschwerpunkt : Lehrwerkkritik, Lehrwerkverwendung, Lehrwerkentwicklung, vol. 2. pp. 83-105.

Morkötter, S. (2009). « Savoir apprendre im frühen Sekundarstufenunterricht - Strategien in den Lehrwerken English G 2000 und À plus ! der Klassen 5 und 6 ». Französisch heute, vol. 40, no 2 . pp. 53-59.

North, B. \& Piccardo, E. (2016). « Developing illustrative descriptors of aspects of mediation for the Common European Framework of Reference (CEFR) ». Consulté en avril $2017:$ https:// www.researchgate.net/ publication/303711981_Developing_illustrative_descriptors_of_aspects_of_mediation_for_the_Common_European_Framework_of_Re

Piccardo, E. (2012). « Médiation et apprentissage des langues : pourquoi est-il temps de réfléchir à cette notion? ». Études de Linguistique Appliquée, vol. 167. pp. 285-297.

Puren, C. (2011). « Une technologie ancienne peut-elle être rénovée ? Le cas du manuel de langue de spécialité face aux nouveaux enjeux de la perspective actionnelle ». Intervention du 16 juin 2011 à la IXe Rencontre internationale du GERES, Université Grenoble 3, Département LANSAD, 16-17 juin 2011. Consulté en mars 2017 : http://www.christianpuren.com.

Reimann, D. (2013). « Evaluation mündlicher Sprachmittlungskompetenz. Entwicklung von Deskriptoren auf translationswissenschaftlicher Grundlage ». In Reimann, D. \& Rössler, A. (dir.). Sprachmittlung im Fremdsprachenunterricht. Tübingen : Narr. pp. 194-226.

Reimann, D. \& Rössler, A. (dir.) (2013). Sprachmittlung im Fremdsprachenunterricht. Tübingen : Narr. Schädlich, B. (2016). « Médiation linguistique et didactique du plurilinguisme et du pluriculturalisme ». In Medhat-Lecocq, H., Negga, D. \& Szende, T. (dir.), Traduction et apprentissage des langues. Entre médiation et remédiation. Paris : Éditions des Archives Contemporaines. pp. 81-91.

Schöpp, F. (2013). Sprachmittlung Französisch. Stuttgart : Ernst Klett Verlag.

Schröder-Sura, A. \& Melo-Pfeifer, S. (2017). «L'intégration des approches plurielles dans les manuels de langues étrangères en Allemagne : tendances et défis ». Beacco, J-C. \& Tremblay, Ch. (dir.), Plurilinguisme et éducation, Volume 2. Paris: L'Observatoire Européen du Plurilinguisme. pp. 89-104.

Stathopoulou, M. (2015). Cross-Language Mediation in Foreign Language Teaching and Testing. Bristol: Multilingual Matters. 
Tesch, B. (2014). « Standards, Bildung, Abitur : Die fortgeführte Fremdsprache vor neuen Herausforderungen ». Die neueren Sprachen. Neue Bildungsstandards und Abitur, Jb.3. Braunschweig : Diesterweg. pp. 11-26.

Zarate, G., Gohard-Radenkovic, A., Lussier, D. \& Hermine, P. (2003). Médiation culturelle et didactique des langues. Strasbourg: Editions du Conseil de l'Europe.

\section{NOTES}

1. Des listes de descripteurs spécifiques viennent d'être publiées. La version française est annoncée pour l'automne 2018. Cf. http://www.coe.int/en/web/common-european-frameworkreference-languages.

2. On peut imaginer par exemple une activité dans laquelle des apprenants de français en Allemagne reçoivent un texte en italien ou en espagnol dont ils doivent restituer le contenu en allemand (p.ex. Schöpp, 2013 : 54f). En même temps, on renforce l'intérêt de l'apprenant pour le français.

3. Pour une discussion autour de ces élargissements possibles, cf. Candelier \& Schröder-Sura, 2015.

4. Elle se distingue ainsi du terme plus général de «médiation » employé pour la résolution des conflits.

5. «Tâches de médiation sont celles qui impliquent, de la part de l'apprenant, le passage d'information d'une langue à une autre avec un but communicatif (...). Plus spécifiquement, quand les usagers linguistiques sont appelés à accomplir une tâche de médiation, ils doivent tout d'abord comprendre l'information inclue dans le texte dans une langue donnée et plus transmettre une partie de l'information dans une autre langue, et ceci de façon appropriée au contexte de la situation. Dans d'autres mots, ils doivent sélectionner quels messages servent le propos d'une rencontre communicative spécifique, pour les transférer de façon adéquate et correcte.»

6. Nous distinguons les notions de Langue Maternelle et de Langue de Scolarisation pour bien mettre en évidence l'hétérogénéité des répertoires linguistiques présents en classe. Ainsi, si l'allemand peut bien être considéré comme LM de quelques apprenants, il est «la langue de l'école » pour une partie de la population scolaire.

7. «Dans les cas où la médiation a été incluse dans les curriculums et les examens, elle tend à impliquer l'interprétation/la traduction ou le résumé d'un texte - écrit ou oral - d'une langue vers une autre. Pourquoi cette interprétation a-t-elle prévalu?» (notre traduction).

8. «La médiation concerne le rôle de la langue dans des processus comme la création d'un espace et des conditions qui facilitent la communication, la compréhension et/ou l'apprentissage, la construction et la co-construction d'un nouveau sens, et la transmission d'information » (notre traduction).

\section{RÉSUMÉS}

Dans cette contribution, nous analysons les tâches de médiation proposées dans une série de manuels de Français Langue Étrangère publiée en Allemagne. Notre but est de confronter ces 
tâches aux définitions de la médiation circulant en Allemagne dans trois types de documents : les documents du Conseil de l'Europe (plus précisément le CECRL et le CARAP), les programmes officiels pour les langues vivantes et les textes théoriques (ré-) interprétant la notion de médiation et proposant des outils de travail permettant sa mise en œuvre pédagogique (notamment en termes d'évaluation). Nous analyserons l'évolution de la présence des tâches de médiation tout au long des quatre manuels d'une série intitulée «A plus ! Nouvelle édition » ainsi que la nature des tâches proposées, notamment en termes de langues ciblées et de situations communicatives exolingues traitées. Nous concluons sur le besoin de diversifier et de complexifier les tâches, ainsi que sur celui de s'appuyer sur une analyse plus fine des contextes et des situations de médiation.

In this contribution, we analyze mediation tasks present in a textbook series for the teaching and learning of French as a foreign language in Germany. We aim at comparing those tasks to the definition of "mediation" currently being spread in Germany in three types of documents: those of the Council of Europe (more specifically the CEFR and the FREPA), the official programs for modern languages and the theoretical accounts (re)interpreting the notion of mediation and proposing pedagogical approaches regarding its integration in the classroom. We will analyze the evolution of the presence of mediation tasks along four schoolbooks of a series called "A plus! Nouvelle édition", as well as the characteristics of those tasks, namely in terms of languages and targeted exolingual communicative situations. Our conclusions point towards the need for the diversification and complexification of tasks, as well as towards the need for a more detailed analysis of contexts of mediation situations in order to inform a more holistic task construction.

\section{INDEX}

Mots-clés : médiation, communication exolingue, approches plurielles, manuels de langues étrangères

Keywords : mediation, exolingual communication, pluralistic approaches, foreign languages textbooks

\section{AUTEURS}

\section{SÍLVIA MELO-PFEIFER}

Universität Hamburg (Allemagne)

Sílvia Melo-Pfeifer a obtenu son doctorat en Didactique des Langues à l'Université de Aveiro (Portugal) et elle est actuellement professeur de didactique des langues romanes (français et espagnol) à l'Université de Hambourg (Allemagne). Elle fait également partie du CIDTFF (Centre de Recherche en Didactique et Technologie dans la Formation de Formateurs), de l'Universidade de Aveiro (Portugal). Elle s'intéresse principalement aux approches plurielles dans l'enseignement-apprentissage des langues et dans la formation initiale, tout particulièrement à l'intercompréhension, ainsi qu'au fonctionnement de la communication plurilingue notamment lorsqu'elle est médiatisée par ordinateur. silvia.melo-pfeifer[at]uni-hamburg.de

\section{ANNA SCHRÖDER-SURA}

Universität Rostock (Allemagne)

Anna Schröder-Sura est enseignante en didactique des langues romanes à l'Université de 
Rostock (Allemagne). Ses domaines de spécialité et de recherche sont la didactique du plurilinguisme, les approches plurielles, l'intercompréhension des langues romanes pour germanophones et la formation des enseignants. Elle a participé à plusieurs projets de recherche et de formation, en particulier à l'élaboration et la diffusion du Cadre de référence pour les approches plurielles des langues et des cultures (CARAP).

anna.schroeder-sura[at]uni-rostock.de 